\title{
MODELO CALGARY DE AVALIAÇÃO FAMILIAR: AVALIAÇÃO DE FAMÍLIAS COM INDIVÍDUOS ADOECIDOS DE TUBERCULOSE
}

\author{
Thais Cristina Flexa Souza1, Adriana Borges Melo², Carla Monique Lavareda Costa 3 , Jacira Nunes Carvalho ${ }^{4}$
}

Objetivo: avaliar famílias com membros em tratamento de tuberculose por meio do Modelo Calgary de Avaliação de Família (MCAF). Metodologia: estudo do tipo descritivo-exploratório no qual participaram duas unidades familiares possuindo um membro em tratamento de tuberculose, ambas cadastradas em uma Estratégia Saúde da Família em Belém, no Pará. Para a aplicação do Modelo, foi realizada a técnica de entrevista semiestruturada e uso de genograma e ecomapa. Resultados: após a avaliação de família realizada conforme Modelo Calgary de Avaliação de Família, resultaram duas famílias, sendo uma monoparental, liderada pela mulher, e a outra alargada; em ambas, o membro adoecido é o provedor de questões econômicas e as familias, pesquisadas possuem relacionamentos e vínculos com algumas instituições de saúde, religiosas, escolares e até grupos de apoio social. Conclusões: o Modelo Calgary de Avaliação de Família mostrou ser eficaz como uma nova estratégia de avaliação, contribuindo para o planejamento de cuidados de famílias em qualquer nível de atenção à saúde.

Descritores: Enfermagem de família, Atenção Primária à Saúde, Serviços de Assistência Domiciliar.

\section{CALGARY MODEL OF FAMILYEVALUATION: EVALUATION OF FAMILIES WITH INDIVIDUALSSICKENED WITHTUBERCULOSIS}

Objective: Evaluating family members in treatment of Tuberculosis through the Calgary Model of Family Evaluation (CMFE). Methodology: Descriptive-exploratory type study, in which two family units having a member in treatment of tuberculosis participated, both registered in a Family Health Strategy, Belém, Pará. For the application of the Model, the technique of semistructured interview was perfomed and utilization of genogram and eco-map. Results: After the evaluation of the family was performed according to Calgary Model of Family Evaluation resulted in two families, one being a mono-parental headed by the woman and the other expended, in both cases the sick member is the provider of economic matters and the families researched have ties and relationships some health institutions, religious, school and even social support groups. Conclusions: The Calgary Model of Family Evaluation shows to be effective as a new evaluation strategy, contributing to the family planning care at any level of health care.

Descriptors: Family Nursing, Primary Health Care, Home Care Services.

\section{MODELO CALGARY DE EVALUACIÓN DE LA FAMILIA: EVALUACIÓN DE FAMILIA CON INDIVIDUOS ENFERMADOS DE TUBERCULOSIS}

Objetivo: Evaluar familia de miembros en el tratamiento de la tuberculosis por medio del Modelo Calgary de Evaluación de la Familia (MCEF). Metodología: Estudio de exploración de tipo descriptivo, participaran dos unidades familiares que tienen un elemento en el tratamiento de la tuberculosis, ambas registradas en una Estrategia de Salud Familiar, Belém, Pará. Para a aplicación do Modelo, fue usada la técnica de entrevista semiestructurada y utilización del genograma y eco-mapa. Resultados: Después de la evaluación de la familia realizado de acuerdo con Modelo Calgary de Evaluación de la Familia resultaron dos familias, una siendo mono parental liderada por la mujer y otra extendida, en los dos el miembro enfermo es el proveedor de los recursos económicos y las familias investigadas tienen relaciones y vínculos con algunas instituciones de salud, religiosas y escolares, hasta grupos de apoyo social. Conclusiones:El Modelo Calgary de Evaluación de la Familia se muestreó ser eficaz como una nueva estrategia de evaluación contribuyendo para el planificación de cuidados familiares en cualquier nivel de atención a la salud.

Descriptores: Enfermería de la familia, Atención Primaria de Salud, Servicios de Atención de Salud a Domicilio.

${ }^{1}$ Enfermeira. Discente do Mestrado. Universidade Federal do Pará (PPGENF/UFPA). Membro do Grupo de Pesquisa EPOTENA. E-mail: thaisflexxa@gmail.com ${ }^{2}$ Enfermeira. Membro do Grupo de Pesquisa EPOTENA.

${ }^{3}$ Enfermeira. Membro do Grupo de Pesquisa EPOTENA.

${ }^{4}$ Enfermeira. Doutora em Enfermagem. Lider do Grupo de Pesquisa EPOTENA. UFPA. 


\section{INTRODUÇÃO}

A tuberculose (TB) é uma doença infecciosa e transmissivel, fortemente favorecida pela precariedade das condições de vida que afeta um grande número de pessoas no mundo, sendo um problema de saúde pública, apesar de seu prognóstico de cura. Anualmente, são notificados, aproximadamente, nove milhões de casos em todo o mundo, de acordo com dados estimados em 2013 pela Organização Mundial da Saúde (OMS), levando mais de um milhão de pessoas a óbito, correspondendo cerca de $80 \%$ dos casos em 22 países. A Índia, a China e a África do Sul são os países com maior incidência da doença e, mundialmente, o Brasil no ranking de 2015 ocupa o 160 lugar em número absoluto de $\operatorname{casos}^{(1)}$.

Em 2014, o Brasil registrou 67.966 casos novos de tuberculose. Ao longo dos anos, observa-se redução do coeficiente de incidência, passando de 41,5/100 mil hab. em 2005, para 33,5/100 mil hab. em 2014, o que corresponde a uma redução média de 2,3\% ao ano, nesse período. A taxa de incidência da tuberculose relacionada à distribuição por região foi: $45,16 \%$ na região Norte, $36,39 \%$ na região Nordeste, $40,15 \%$ na região Sudeste, $32,30 \%$ na região Sul e região Centro-Oeste com $24,66 \%$, com um total de $37,28 \%$ na taxa de incidência. Em relação à região Norte, o estado do Pará foi identificado como - $4^{\circ}$ estado, com uma incidência de tuberculose de $44,96 \%{ }^{(2)}$.

Diante dessa realidade, as políticas públicas de controle dessa endemia recriam formas de melhor atender à população na esfera da Atenção Primária. É criado, então, o Programa Saúde da Família, hoje Estratégia Saúde da Familia (ESF), que tem em vista o serviço da atenção centrada na família e comunidade( ${ }^{(3)}$. Assim, a ESF é uma modalidade que incorpora práticas preventivas, educativas e curativas nos grupos mais vulneráveis, desenvolvendo suas ações mais próximas da população, podendo estabelecer vínculos entre profissionais de saúde e família ${ }^{(4)}$. Contudo, no caso específico da tuberculose, é essencial que os profissionais de saúde também conheçam os possiveis contatos para que possam, além de fazer orientações sobre a doença e o tratamento, realizar medidas de prevenção(5,6).

Assim, são colocados novos desafios em relação à enfermagem, como líder dessa equipe; entre esses desafios, estão desenvolver as habilidades para a prática do cuidado com a família como uma unidade complexa, composta por uma diversidade de elementos que dividem o mesmo espaço e mantém relações afáveis ou não(7).

Desse modo, para se trabalhar a enfermagem de família, o Modelo Calgary de Avaliação de Família (MCAF) foi proposto e implantado por Wright e Leahey, pesquisadoras da Universidade de Calgary, no Canadá. Tal modelo tratase de um referencial metodológico que permite analisar a família como um sistema, por meio do diagnóstico de seus problemas de saúde, seus recursos potenciais para enfrentar os problemas e os suportes sociais comunitários disponíveis. Para a avaliação da família, são realizadas entrevistas semiestruturadas, utilizando-se instrumentos como: o genograma (desenho da família) e o ecomapa (desenho das relações das famílias e grupos externos). Por meio desses instrumentos, pode-se verificar as estruturas internas e externas da familia, os membros que a compõem, o vinculo afetivo entre eles e o contexto familiar no qual estão inseridos ${ }^{(7)}$.

O MCAF surge como uma possibilidade para essa prática. A aplicação desse modelo permite realizar a análise familiar, levando em consideração os principais aspectos de sua estrutura, desenvolvimento e funcionamento, com a finalidade de ajudar a entender a importância do cuidado em família, propiciando uma articulação mais eficiente aos serviços de saúde, tendo em vista que a doença não pode ser considerada um caso isolado de determinados membros. Esse modelo é uma ferramenta que permite a enfermagem avaliar e investigar famílias em diversos contextos, facilitando o fortalecimento do vínculo entre profissional-paciente-família(8-10).

Diante do exposto, é importante ressaltar que ainda são escassos os estudos que enfocam enfermagem de família, principalmente trazendo o MCAF. Por isso, torna-se um tema pertinente para investigação. $O$ estudo tem como objetivo avaliar familias de indivíduos em tratamento de tuberculose por meio do MCAF.

\section{METODOLOGIA}

Estudo do tipo descritivo-exploratório, realizado por acadêmicas de enfermagem da Universidade Federal do Pará, no qual participaram duas unidades familiares, ambas cadastradas em uma ESF, Belém, Pará.

Aescolha dessasfamilias ocorreu de formaintencional, com pelo menos um membro em tratamento de tuberculose e que tivessem endereço correto no prontuário. Os pesquisadores realizaram visitas a cada família para conhecer a realidade de moradia e saneamento e melhor compreender as relações e comportamentos de vida e cuidado à saúde de seus membros. Para a aplicação do MCAF, foi realizada uma entrevista semiestruturada, constituída de tópicos norteadores, baseada no diagrama ramificado do MCAF, que aborda aspectos de avaliação estrutural, de desenvolvimento e funcional, com elaboração de genograma e ecomapa da família. As famílias entrevistadas assinaram o Termo de Consentimento Livre e Esclarecido (TCLE), autorizando a participação no estudo e a garantia do anonimato foi preservada.

O genograma é um diagrama que detalha a estrutura e o histórico familiar. Geralmente, incluem-se, pelo menos, três gerações e os membros da família são colocados em séries horizontais, que significam linhagens de geração. O ecomapa é um diagrama que representa a visão geral da situação da família, retratando as relações de educação ou conflitos 
entre família e o mundo. Coloca-se o genograma da família dentro de um círculo central e fazem-se círculos externos representando pessoas, órgãos ou instituições no contexto familiar ${ }^{(4)}$

A categoria estrutural compreende a estrutura da família. Três aspectos da estrutura familiar podem ser examinados, como: elementos internos (composição da família, gênero, orientação sexual, ordem de nascimento, subsistemas e limites), elementos externos (familia extensa e sistemas mais amplos) e contexto (etnia, raça, classe social, religião e ambiente) $)^{(4)}$

Em relação à categoria de desenvolvimento, essa se refere à transformação progressiva da história familiar durante as fases do ciclo de vida: sua história, o curso de vida, o crescimento da família, o nascimento, a morte e suas subcategorias são classificadas em estágios, tarefas e vínculos ${ }^{(4)}$.

Quanto à categoria funcional, essa se refere ao modo como os individuos da familia interagem. Podem ser explorados dois aspectos: o funcionamento instrumental, que se refere às atividades da vida cotidiana, e o funcionamento expressivo, que diz respeito aos estilos de comunicação, solução de problemas, papéis, crenças, regras e alianças ${ }^{(4)}$.

A coleta de dados foi agendada conforme a disponibilidade dos membros das familias, a fim de garantir a sua participação. $\mathrm{Na}$ fase de análise, os dados foram descritos conforme a estrutura, o desenvolvimento e a funcionalidade da unidade familiar estudada.

Esta pesquisa traz riscos mínimos ao entrevistado, e a coleta de dados somente ocorreu mediante a autorização da Secretaria Municipal de Saúde (SESMA) e do Comitê de Ética com Seres Humanos do Instituto de Ciências da Saúde, com número do parecer 961.277, data da relatoria 26/01/2015(11).

\section{RESULTADOS}

As avaliações das familias Fl e F2 foram feitas, no próprio domicilio, na presença de todos os membros. A avaliação de família foi realizada de acordo com a metodologia do MCAF (estrutura, desenvolvimento e funcionalidade).

A primeira familia ( $F l)$ é composta por mãe e filho. A mãe (PPl) é uma senhora de 72 anos, aposentada, viúva, cuida dos afazeres domésticos e é a cuidadora principal. O filho (PP2) feminino.

PPl atualmente está em tratamento de tuberculose; além disso, possui problemas oftalmológicos, na coluna vertebral e sinusite crônica. PP1 e PP2, quando necessário, utilizam o serviço de saúde local, a ESF. Segundo informações de PPl, no momento, o filho não possui nenhum problema de saúde, porém informa ser este usuário de drogas, o que dificulta as relações entre eles. PPl é evangélica; relata que seus laços de amizade são na maioria com participantes da igreja, os quais the dão apoio emocional e companhia social; comentou, ainda, que sua relação com a vizinhança é pacífica. Em relação aos outros filhos, PPl relatou que todos estão casados e moram distante dela, o que the causa uma grande tristeza. Em sua família, nunca existiu o hábito de reuniões familiares, sendo o encontro pessoal com esses membros muito raro, ocorrendo apenas em datas comemorativas; o contato via telefone também é pouco frequente, devido a suas baixas condições financeiras.

A segunda familia (F2) compõe-se de cinco membros, sendo três adultos e duas crianças. O familiar doente $(\mathrm{Pl})$ é do sexo masculino casado, 33 anos, extrativista; sua esposa (P2) é do lar, 31 anos, e a participante (P3)- cunhada do familiar adoecido é solteira, tem 28 anos, desempregada. $\mathrm{O}$ casal (PI e P2) concebeu duas filhas: uma tem 12 anos (P4), estudante, e a outra tem 3 anos (P5). Esse grupo familiar reside em casa própria de alvenaria, com fossa séptica e saneamento básico. A água para consumo é distribuida por sistema de abastecimento público. Todas as pessoas residentes nesse núcleo familiar, quando necessário, utilizam o serviço de saúde local, a ESF. Não relataram problemas de saúde em outros membros da família. Verificou-se que existe bom relacionamento na F2, sendo que, após o diagnóstico da tuberculose, o vínculo ficou ainda mais forte entre eles. Afirmam, ainda, que todos os membros da família são evangélicos e que se relacionam bem na igreja. Relatam que a relação com a vizinhança é muito boa.

Nas ilustrações abaixo (Quadro 1, Quadro 2, Figura 1, Figura 2), ressaltam-se algumas características das famílias, segundo o MCAF:

Quadrol - Família 1 (Fl) constituída por 2 membros. Belém, PA Brasil, 2016. que reside com a mãe é o primeiro de uma prole de quatro filhos (4). Residem em casa própria de alvenaria, a qual possui fossa séptica e saneamento básico. A água para consumo é distribuída por sistema de abastecimento público. PPl e seu marido conceberam quatro filhos, sendo os dois primeiros do sexo masculino e dois últimos, do sexo

\begin{tabular}{|c|c|c|c|c|c|c|}
\hline \multicolumn{7}{|l|}{ FAMÍLIA 1} \\
\hline Membro & Sexo & Idade & Ocupação & $\begin{array}{l}\text { Saneamento } \\
\text { básico }\end{array}$ & $\begin{array}{l}\text { Problemas de } \\
\text { saúde referidos }\end{array}$ & Relação social \\
\hline $\begin{array}{l}\text { Participante } 1 \\
\text { (PPl) }\end{array}$ & $\mathrm{F}$ & 72 & $\begin{array}{l}\text { Aposen- } \\
\text { tada }\end{array}$ & Sim & $\begin{array}{l}\text { Tuberculose, } \\
\text { problemas de } \\
\text { visão, coluna e } \\
\text { sinusite crônica }\end{array}$ & $\begin{array}{l}\text { Vizinhança } \\
\text { e igreja }\end{array}$ \\
\hline $\begin{array}{l}\text { Participante } 2 \\
\text { (PP2) }\end{array}$ & $M$ & 38 & $\begin{array}{l}\text { Desempre- } \\
\text { gado }\end{array}$ & Sim & $\begin{array}{l}\text { Dependência } \\
\text { química }\end{array}$ & $\begin{array}{l}\text { Vizinhança } \\
\text { e grupo de } \\
\text { usuário de } \\
\text { drogas }\end{array}$ \\
\hline
\end{tabular}


FIGURA 1 - Ecomapa da Familia 1 (F1). Belém, PA, Brasil, 2016.

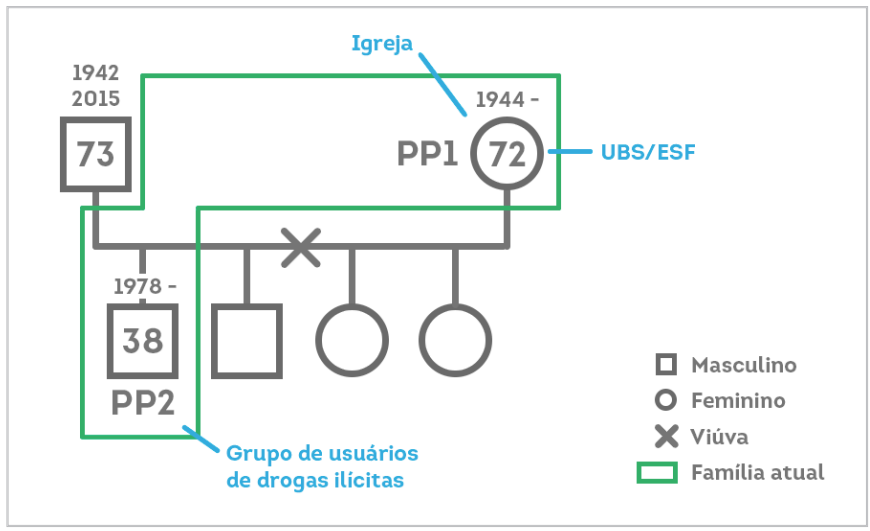

Quadro 2 - Família 2 (F2) constituída por 5 membros. Belém, PA, Brasil, 2016.

\begin{tabular}{|c|c|c|c|c|c|c|}
\hline \multicolumn{7}{|l|}{ FAMÍLIA 1} \\
\hline Membro & Sexo & Idade & Ocupação & $\begin{array}{l}\text { Saneamento } \\
\text { básico }\end{array}$ & $\begin{array}{l}\text { Problemas de } \\
\text { saúde referidos }\end{array}$ & Relação social \\
\hline $\begin{array}{l}\text { Participante l } \\
\text { (PPl) }\end{array}$ & $M$ & 33 & Extrativista & Sim & Tuberculose & $\begin{array}{l}\text { Vizinhança } \\
\text { e igreja }\end{array}$ \\
\hline $\begin{array}{l}\text { Participante } 2 \\
\text { (PP2) }\end{array}$ & $\mathrm{F}$ & 31 & Dona de casa & Sim & - & $\begin{array}{l}\text { Vizinhança } \\
\text { e igreja }\end{array}$ \\
\hline $\begin{array}{l}\text { Participante } 3 \\
\text { (P3) }\end{array}$ & $\mathrm{F}$ & 28 & Desempregada & Sim & - & $\begin{array}{l}\text { Vizinhança } \\
\text { e igreja }\end{array}$ \\
\hline $\begin{array}{l}\text { Participante } 4 \\
\text { (P4) }\end{array}$ & $\mathrm{F}$ & 12 & Estudante & Sim & - & $\begin{array}{l}\text { Vizinhança } \\
\text { e igreja }\end{array}$ \\
\hline $\begin{array}{l}\text { Participante } 5 \\
\text { (P5) }\end{array}$ & $\mathrm{F}$ & 3 & - & Sim & - & $\begin{array}{l}\text { Vizinhança } \\
\text { e igreja }\end{array}$ \\
\hline
\end{tabular}

FIGURA 2 - Ecomapa da Família 2 (F2). Belém, PA, Brasil, 2016.

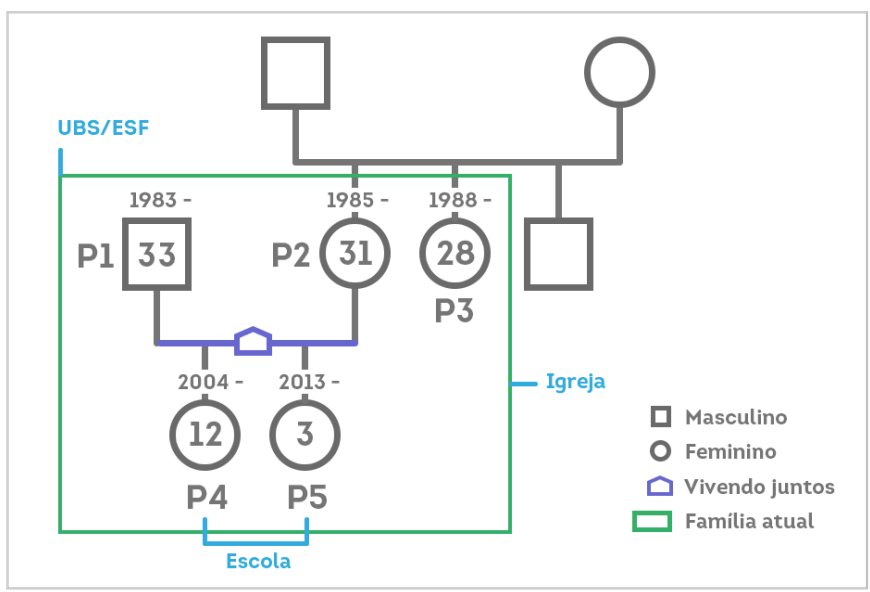

A familia como unidade caracteriza-se, essencialmente, pelas inter-relações estabelecidas entre os seus membros, em um contexto específico de organização, estrutura e funcionalidade, não se levando em consideração o número de membros ${ }^{(12)}$. Entende-se que a quantidade de membros na $\mathrm{Fl}$ e

\section{DISCUSSÃO} F2 não influencia no vínculo que possa ocorrer entre eles.
Nas duas familias, foi possivel verificar a condição das relações interpessoais entre seus membros, ficando patente a dificuldade diante de pessoas adidas à drogadição. Podese entender essa dificuldade, dada a intolerância de pessoas usuárias de drogas às orientações ou intervenções de terceiros.

É na família que os indivíduos iniciam seus processos de formação da personalidade, a qual é importante na recuperação do usuário. Passa a ser a sua ligação fundamental com a sua comunidade, devendo ser incluída, acolhida e cuidada nos serviços de saúde. A família deve ser parceira no tratamento, como rede de relação que dá suporte ao usuário para enfrentar as dificuldades cotidianas, advindas das drogas ${ }^{(13)}$.

A interdependência entre a saúde da família, como unidade funcional, e a saúde dos seus membros pressupõe que os cuidados de saúde serão tanto mais eficazes quanto maior a ênfase no sistema familiar ${ }^{(14)}$. Logo, pode-se entender que a coresponsabilização de cuidados entre os membros de uma família é essencial para o sucesso de qualquer tratamento e para o bem estar do grupo familiar(15). Por exemplo, na F2, os membros são mais próximos, com vínculos fortalecidos, o que pode aumentar as chances de $\mathrm{Pl}$ não abandonar o tratamento de tuberculose: diferentemente, a $\mathrm{Fl}$ tem vínculos frágeis e, como já dito anteriormente, a possibilidade de não adesão ao tratamento é maior.

Os genogramas e ecomapas permitem a visualização de cada uma das famílias como uma unidade, por conseguinte. focada na interação entre seus membros ${ }^{(4)}$. Nesse sentido, o cuidado realizado no domicilio exige, da enfermagem, romper barreiras para conhecer a família no seu cotidiano, respeitar suas crenças, culturas e valores ${ }^{(15)}$. Com essas ferramentas, teve-se como avaliar Fl e F2, conhecendo mais um pouco sobre seus laços afetivos, podendo melhorar e direcionar o tratamento com orientações mais adequadas a cada família.

Os profissionais devem usar seus conhecimentos sobre cada familia, para juntos pensarem e programarem a melhor assistência possivel. Dessa forma, é papel do enfermeiro em saúde da família reconhecer os fatores sociais, econômicos, culturais vivenciados e interagir com situações que apoiem a integridade familiar ${ }^{(16)}$. Percebeu-se, também, que a visita domiciliar de um enfermeiro ajudou Fl e F2 a esclarecer suas dúvidas e desmistificar seus tabus sobre a tuberculose. Para o enfermeiro, isso contribuiu para realizar um plano de cuidados 
voltado para as condições socioeconômicas da família.

Os fatores culturais podem causar ou contribuir para o surgimento de problemas de saúde, assim como podem protegê-los. As condições de saúde estão intimamente relacionadas com o meio em que a pessoa está inserida (16). Assim, destaca-se que Pl da F2 é extrativista vegetal, desenvolvendo seu trabalho em comunidades próximas à área rural. Por ser essa uma atividade laboral que exige força e maior desgaste físico, e ele ser o único que possui atividade remunerada, a recuperação da sua saúde pode ficar mais dificultada.

É imprescindivel avaliar o desempenho dos serviços de controle da tuberculose nos diferentes cenários e em todas as suas dimensões, inclusive em relação ao enfoque familiar, visto que a assistência à saúde deve ser centrada na família. A avaliação das necessidades de saúde deve considerar - contexto familiar e a exposição a ameaças à saúde de qualquer ordem, além dos recursos familiares limitados ${ }^{(17,18)}$. Com a utilização do MCAF, nas duas famílias, foi possivel ver a tuberculose em dois cenários diferentes, uma família com laços fortalecidos e outra com vínculos fragilizados, e percebeu-se que a adesão da familia é extremamente importante para o sucesso do tratamento.

\section{CONCLUSÃO}

Por meio da descentralização das ações em saúde e reestruturação dos serviços, a ESF assume um papel essencial no controle da tuberculose, com chances de tornar-se protagonista no combate à doença, uma vez que, durante a visita domiciliar, pode-se criar vínculos com a família e pessoas da comunidade, facilitando a adesão ao tratamento.

Dessa maneira, a assistência à familia como unidade de cuidado implica em conhecer como cada família cuida e identifica suas forças, suas dificuldades e seus esforços para partilhar as responsabilidades.

O combate à tuberculose não deve se limitar apenas aos aspectos patológicos; faz-se necessária uma visão ampliada e humanizada por parte da equipe multiprofissional que extrapole os muros das unidades de saúde e se articule com outros setores que possam dar suporte social ao doente e sua familia, oferecendo suporte ao tratamento e combate à doença.

Portanto, a enfermagem de familia é importante para ser o elo de referência entre o serviço de saúde e a família, mostrando-se bastante eficaz ao utilizar o MCAF como uma nova estratégia de avaliação, contribuindo para o planejamento de cuidados de famílias em qualquer nível de atenção à saúde.

\section{REFERÊNCIAS}

1 Pereira JC, Silva MR, Costa RR, Guimarães MDC, Leite ICG. Perfil e seguimento dos pacientes com tuberculose em municipio prioritário no Brasil. Rev Saúde Pública. 2015; 49(6): 1-12.

2 Datasus. Brasilia: Ministério da Saúde. Sistema de Informação de Agravos de Notificação. Disponivel em:http://tabnet datasus.gov.br/cgi/deftohtm. exe?idb2012/d0202.def

3 Brito EWG Silva AKF, Teixeira GGA et al Organização do cuidado à tuberculose na atenção básica do Rio Grande do Norte. Rev enferm UFPE on line 2015; 9(Supl. 6):8643-52. DOI: 10.5205/reuol.7061-61015-5SM0906supl201503.

4 Wright L, Leahey M.Enfermeiras e Familias: Um guia para a avaliação e intervenção na familia.São Paulo: Roca; 2012.

5 Cecilio HPM, Higarashi IH, Marcon SS. Opinião dos profissionais de saúde sobre os serviços de controle da tuberculose. Actapaul.enferm. 2015; 28(1): $19-25$.

6 Coutinho LRP, Barbieri AR, Santos MLM. Acolhimento na Atenção Primária à Saúde: revisão integrativa. Saúde Debate, 2015; 39(105): 514-524.

7 Baia RSM, Gonçalves LHT, Oliveira MFV. Wright LM, Leahey M. Enfermeiras e familias: guia para avaliação e intervenção na familia. 5a ed. São Paulo (SP): Roca; 2012. Rev Rene. set-out; 15(5):904-5.

8 Maciel ELN, Sales CMM. A vigilância epidemiológica da tuberculose no Brasil: como é possivel avançar mais? Epidemiol. Serv. Saúde, Brasilia, 2016 25(1): 175-178.

9 Fernandes CS, Gomes JAP, Martins MM, Gomes BP, Gonçalves LHT. A Importância das Familias nos Cuidados de Enfermagem: Atitudes dos Enfermeiros em Meio hospitalar. Rev. Enf. Ref. 2015: (7): 21-30.

10 Rodrigues FA, Costa SFG, Fernandes MA, Zaccara AAL, Duarte MCS,
Andrade CG. Produção científica acerca do modelo Calgary de avaliação da familia: um estudo bibliometrico Rev. pesqui. cuid fundam. (Online) 2015 7(3): 3063-3075.

11 Resolução № 466 do Conselho Nacional de Saúde, de 12 de dezembro de 2012 (BR) [Internet]. Aprova as diretrizes e normas regulamentadoras de pesquisas envolvendo seres humanos. Diário Oficial da União. 12 dez 2012 [acesso em: 06 jul 2016]. Disponivel em: http://bvsms.saude.gov.br/bvs/ saudelegis/cns/2013/res0466_12_12_2012.html

12 Gomes MFP, Fracolli RA, Machado BC. Atenção domiciliar do enfermeiro na estratégia saúde da familia. O Mundo da Saúde 2015; 39(4):470-475.

13 Alvarez SQ, Gomes GC, Xavier DM. Causas da dependência química e suas consequências para o usuário e a familia. Rev enferm UFPE on line. 2014; 8(3):641-8

14 Clementino FS, Miranda FAN. Tuberculose: acolhimento e informação na perspectiva da visita domiciliária. Rev. Enf. UERJ 2015. 23(3): 350-354.

15 Monteiro GRSS, Moraes JCO, Costa SFG, Gomes BMR, França ISX Oliveira RC. Aplicação do Modelo Calgary de Avaliação Familiar no contexto hospitalar e na atenção primária à saúde: Revisão integrativa. Aquichan. 2016; 16 (4): 487-500.

16 Caçador BS, Brito MJM, Moreira DA, Rezende LC, Vilela GS. Ser enfermeiro na estratégia de saúde da familia: desafios e possibilidades. REME rev.Min. enferm,2015. 19(3): 612-626.

17 Santos DS, Tenório EA, Brêda MZ,Mishima SM. Processo saúde/doença e estratégia de saúde da familia: o olhar do usuário. Rev. Latino-Am. Enfermagem 2014; 22(6):918-25.

18 Furlan MCR, Gonzales RIC, Marcon SS. Desempenho dos serviços de controle da tuberculose em municipios do Paraná: enfoque na familia. Rev Gaúcha Enferm. 2015:36(esp): 102-10. 